\title{
Towards a Mixed-Reality Interface for Mind-Mapping
}

Philippe Giraudeau

Inria Bordeaux Sud-Ouest

200 Avenue de la Vieille Tour,

33405, Talence

philippe.giraudeau@inria.fr

Martin Hachet

Inria Bordeaux Sud-Ouest

200 Avenue de la Vieille Tour,

33405, Talence

martin.hachet@inria.fr

\begin{abstract}
In this work, we have explored an approach based on the

hybridisation of physical and digital content for

mind-mapping activities at schools. Based on the

literature in the fields of cognitive science and $\mathrm{HCl}$, we have designed a mixed-reality (MR) interface called

Reality-Map. We conducted a pilot study with 11 participants suggesting that learning and manipulating information about the brain and their cognitive functions could be improved by the use of such a MR interface compared to a traditional WIMP interface.
\end{abstract}

Author Keywords

Mixed-Reality interface, Learning, Mind-Mapping

\section{ACM Classification Keywords}

H.5.m [Information interfaces and presentation (e.g.,

$\mathrm{HCl}$ )]: Miscellaneous.

\section{Introduction}

The wide majority of digital applications are mainly based on WIMP (Windows, Icons, Menus and Pointing)

interfaces, controlled by way of mice and keyboards. Such interfaces have shown great benefits for web browsing, text editing, and so on. On the other hand, they are limited as soon as collaborative interactions or hands-on activities are required [12]. 
This is particularly true for learning applications [16].

Beyond mice and keyboards, reality-based interaction (Rbl) "increases the realism of interface objects and allow users to interact even more directly with them using actions that correspond to daily practices within the non-digital world" [8]. It also provides hands-on activity and gestures which enhance the user's learning abilities [7]. Good examples of Rbl are Tangible User Interfaces (TUI), which provide physical handles for the manipulation of virtual information [15] and Spatial Augmented Reality (SAR), which complement the real world with digital content [2]. Both SAR and TUI are known to support collaborative activities like learning and problem solving [5].

This paper presents first results of our exploration combining interactions with both the digital and the physical world. More precisely we focused on the design and evaluation of a tangible and augmented user interface dedicated to support learning with the prospect of extending existing usages and activities. In particular, in this work, we were interested in Mind-Mapping activities.

In our approach learners will sit around a table, interacting physically with objects (e.g. sheets of papers or 3D objects) as they would experience it in a non-digital way in a classroom. Those objects can be augmented with digital information thanks to a video projector. Such interfaces will open the way to hybrid interactions, where both physical and virtual can be manipulated in the same hybrid space. Our assumption is that this hybridisation will favour hands-on activities and collaborative learning.

\section{Related Works}

Interactive tabletops allow users to interact with a digital environment on a horizontal surface.
Urp [18] is a system for urban planning that allows users to control a real-time and visually coincident simulation of pedestrian-level wind flow, and control the impact of building's shadows on its surroundings.

In contrast to Urp, which was not developed as an education tabletop, some systems were designed specifically for this purpose. The Tinker Lamp environment is an augmented tabletop interface for training logistics assistants. It was made to understand how to place shelves on a warehouse to make it more efficient $[9,19]$. Results showed that compared to a method with paper and pen, students with The Tinker Lamp were faster and learned more about how to manage a warehouse.

Another example is Hobit [6] which proposes to enhance the learning of Optics with an augmented tabletop that reproduces practicals at university. This interface benefits from both physical manipulation and pedagogical supports that are embedded within the experimentation.

Previous study from Buisine et al [4] compared pen-and-paper interaction with tabletops interface. This interface provided augmentation without tangible objects. Results showed no significant differences on idea production between the two modalities.

The Reactable [10] is a well-known tabletop interface that is able to track the position and orientation of tangible objects on a horizontal surface. This system was initially designed for the creation of musical structures. Its software components, Reactivision, has been used for other purposes such as maps manipulation. 


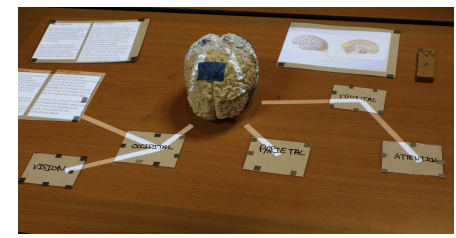

Figure 1: Reality-Map displaying links and digital contents between digital and real objects.

\section{Reality-Map}

We developed a prototype of an augmented and tangible interactive tabletop implementing mind-mapping, named Reality-Map. The system is based on the hybridisation of physical and digital components. Digital images, videos, and texts are projected onto physical sheets of paper that users can manipulate as if they were manipulated standard documents (e.g. printed sheet of paper). Hence,

Reality-Map stands on affordances [13] that make sense for users, and that may favor learning process as suggested by the instructional design literature [17].

With Reality-Map, users can spatially organise the studied content by manipulating the augmented papers in front of them. Then they can build a mind-map by digitally drawing links between the documents. To do so, we use an interactive pen that allows the users to create and remove links between the physical objects. These links are higlighted with straight lines projected between the objects. This very straightforward approach may reduce the mental effort needed to succeed in the task [8, 15].

Beyond the augmented sheets of papers, we also introduced plastic-coated cards allowing users to quickly write with whiteboard markers on them, without the need of keywords and mice. Hence, users can write short texts or sketch ideas, and embed these cards within the mind-map.

In our approach gesture are considered as a mean of communication [15] but also a way of reducing cognitive load by supporting speech [1]. As shown by [14] users tend to try more solutions with physical manipulation and allow them to spend more time exploring when performing problem solving tasks. This physical manipulation is associated with a positive learning gain in some contexts.
The hardware side consists of a Optitrack's Trio composed of three infrared cameras with infrared LEDs and a video-projector placed above the user for the augmentation of the horizontal surface. Physical objects like cards and papers are tracked by Optitrack's Trio thanks to infrared markers placed on each physical object.

Unity3D is used to display the digital documents and the links between them. We use Motive to collect data from Optitrack's Trio and send them to Unity3D. It also allows calibration and visualisation of the markers in the real world.

\section{Evaluation}

We carried out a pilot study to evaluate Reality-Map by comparing it with a WIMP interface called Draw.io ${ }^{1}$.

The assumption of this study was that mixed-reality interfaces based on TUI and SAR are not only good for providing reality-based interactions but may also favour the building of knowledge for learning complex notions. In our case, we chose basic brain functions as the targeted knowledge. Thus, we decided to test several hypotheses:

- H1: Learning scores will be better in the tangible group compared to control group.

- H2: Participants will better recall knowledge learned during the experiment after 5 days with Reality-Map

- H3: Participants will prefer Reality-Map instead of a WIMP counterpart.

Participants were recruited within our laboratory and from the university. To be included in the experiment,

\footnotetext{
${ }^{1}$ http://draw.io
} 


\begin{tabular}{|l|l|l|l|}
\hline Group & \multicolumn{2}{|c|}{ Gender } & Age \\
\hline & Male & Female & $\begin{array}{l}\text { Mean } \\
; \sigma\end{array}$ \\
\hline TG & 4 & 2 & 23,$6 ;$ \\
& & & 2,6 \\
CG & 3 & 2 & 24,$6 ;$ \\
& & & 1,94 \\
\hline
\end{tabular}

participants should have no prior formal knowledge about structures and functioning of the brain (e.g., studied neurosciences, psychology or cognitive science). We recruited twelve participants. Unfortunately, one outlier was excluded from the analysis.

\section{Experimental Design}

For this between-subjects experiment, we randomly split participants into two groups (see Figure 2). The first one was named "Control group", which had to create a mind-map on a computer screen with Draw.io and the second named "Tangible group", which had to create a mind-map on our system.

The procedure was conducted as follows. First, participants answered pretest regarding their general knowledge about brain areas. Then, they were invited to complete two tasks. Depending on their group,

Figure 2: Summary of tested population. TG: Tangible group; CG: Control group

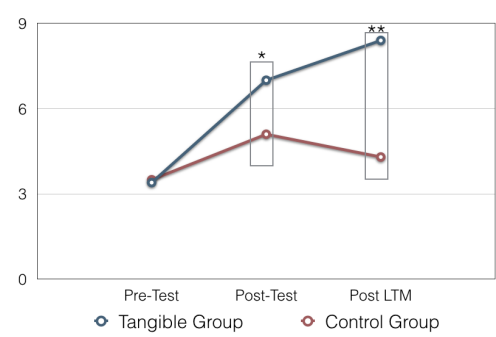

Figure 3: Evolution of the score results between the two groups, the tangible group and the control group. ${ }^{*} \mathrm{p}<.05 ;{ }^{* *} \mathrm{p}<.01$. participants were put either in front of a computer screen with a keyboard and mouse or in front of Reality-Map.

During $10 \mathrm{~min}$, participants discovered and manipulated documents about brain areas. Then, the task consisted in the creation of a mind-map on the following theme:

"Brain structures and their cognitive processes". This task had to be done in $10 \mathrm{~min}$. After these two tasks, participants were invited to answer two user-experience questionnaires, the System Usability Scale (SUS) [3] and the User Experience Questionnaire (UEQ) [11]. Finally, participants had to fill a post-test questionnaire regarding what they learned during the session. 5 days after the experiment, participants were invited to answer a second post-test questionnaires.

\section{Results}

Learning Gain (H1, H2)

To evaluate our results, we conducted a two-way mixed ANOVA with the group as an independent factor, which had two levels (Tangible group and Control group) and time as a repeated measure, which had three levels (pretest, post-test and post-test +5 days).

Analyses showed that participants scored significantly higher in the post-test that they did in the pre-test, $(F=5,256 ; p<.05)$. There is an interaction between the group and the score resulting in a significantly higher score for the tangible group in the post-test than the control group $(F=13.991 ; p<.01)$ (See Figure 3)

Usability (H3)

Finally, we analysed UEQ and SUS results with a t-test on each item's category. Results indicated that neither the 1st SUS nor UEQ showed significant differences between two groups after the two tasks (respectively $[\mathrm{t}=-1.724$, $\mathrm{p}=.119] ;[\mathrm{t}=1.653, \mathrm{p}=1.567])$.

\section{Conclusion}

In this paper, we presented Reality-Map, a MR tool dedicated to the manipulation of both digital and physical objects in the same hybrid space and for the creation a mind-map with the final goal of enhancing the building of knowledge. Results from the pilot study suggest that Reality-Map can enhance learning for this specific task compared to a popular WIMP interface called Draw.io These results tend to validate that interfaces which promote gestures and affordances can enhance learning by reducing the extrinsic cognitive load as describe by Sweller [17]. Among the future work, it will be interesting to compare Reality-Map to a standard non-digital approach. 
Results obtained in the preliminary work will help us to design the next iteration of Reality-Map.

\section{Acknowledgment}

This work is supported by the e-TAC project funded by the e-FRAN program of the French government.

\section{References}

[1] Alibali, M. W., Kita, S., and Young, A. J. Gesture and the process of speech production: We think, therefore we gesture. Language and cognitive processes 15, 6 (2000), 593-613.

[2] Bandyopadhyay, D., Raskar, R., and Fuchs, H. Dynamic shader lamps: Painting on movable objects. In Augmented Reality, 2001. Proceedings. IEEE and ACM International Symposium on, IEEE (2001), 207-216.

[3] Brooke, J., et al. Sus-a quick and dirty usability scale. Usability evaluation in industry 189, 194 (1996), 4-7.

[4] Buisine, S., Besacier, G., Najm, M., Aoussat, A., and Vernier, F. Computer-supported creativity: Evaluation of a tabletop mind-map application. In International Conference on Engineering Psychology and Cognitive Ergonomics, Springer (2007), 22-31.

[5] Dillenbourg, P. What do you mean by collaborative learning. Collaborative-learning: Cognitive and computational approaches 1 (1999), 1-15.

[6] Furió, D., Fleck, S., Bousquet, B., Guillet, J.-P., Canioni, L., and Hachet, M. Hobit: Hybrid optical bench for innovative teaching. In Proceedings of the 2017 CHI Conference on Human Factors in Computing Systems, ACM (2017), 949-959.

[7] Goldin-Meadow, S. How Gesture Promotes Learning Throughout Childhood. 1-6.
[8] Jacob, R. J., Girouard, A., Hirshfield, L. M., Horn M. S., Shaer, O., Solovey, E. T., and Zigelbaum, J. Reality-based interaction: a framework for post-wimp interfaces. In Proceedings of the SIGCHI conference on Human factors in computing systems, ACM (2008), 201-210.

[9] Jermann, P., Zufferey, G., and Dillenbourg, P. Tinkering or sketching: Apprentices' use of tangibles and drawings to solve design problems. In European Conference on Technology Enhanced Learning, Springer (2008), 167-178.

[10] Jordà, S., Geiger, G., Alonso, M., and Kaltenbrunner, $M$. The reactable: exploring the synergy between live music performance and tabletop tangible interfaces. In Proceedings of the 1st international conference on Tangible and embedded interaction, ACM (2007), 139-146.

[11] Laugwitz, B., Held, T., and Schrepp, M. Construction and evaluation of a user experience questionnaire. In Symposium of the Austrian $\mathrm{HCl}$ and Usability Engineering Group, Springer (2008), 63-76.

[12] Marshall, P. Do tangible interfaces enhance learning? Proceedings of the 1st international conference on Tangible and embedded interaction - TEl '07 (2007), 163.

[13] Norman, D. A. Cognitive artifacts. Designing interaction: Psychology at the human-computer interface 1 (1991), 17-38.

[14] Schneider, B., Jermann, P., Zufferey, G., and Dillenbourg, P. Benefits of a tangible interface for collaborative learning and interaction. IEEE Transactions on Learning Technologies 4, 3 (2011), 222-232.

[15] Shaer, O. Tangible User Interfaces: Past, Present, and Future Directions. Foundations and Trends $\AA$ in Human-Computer Interaction 3, 1-2 (2009), 1-137. 
[16] Stanton, D., and Neale, H. . The effects of multiple mice on children's talk and interaction. Journal of Computer Assisted Learning 19, 2 (2003), 229-238.

[17] Sweller, J. Cognitive load theory, learning difficulty, and instructional design. Learning and instruction 4, 4 (1994), 295-312.

[18] Underkoffler, J., and Ishii, H. Urp: a luminous-tangible workbench for urban planning and design. In Proceedings of the SIGCHI conference on
Human Factors in Computing Systems, ACM (1999), 386-393.

[19] Zufferey, G., Jermann, P., Lucchi, A., and Dillenbourg, P. Tinkersheets: using paper forms to control and visualize tangible simulations. In

Proceedings of the 3rd international Conference on Tangible and Embedded interaction, ACM (2009), 377-384. 